\title{
Search for Hidden Sector particles at NA62
}

\author{
Gaia Lanfranchi*广 \\ Laboratori Nazionali di Frascati - INFN \\ E-mail: gaia.lanfranchielnf.infn.it
}

\begin{abstract}
Fixed target experiments are a particularly useful tool in the search of very weakly coupled particles in the MeV-GeV range that could explain the baryon asymmetry of the Universe, the nature of the Dark Matter and the origin of the neutrino masses and oscillations. The NA62 experiment at the CERN SPS is currently taking data to measure the rare decay $K^{+} \rightarrow \pi^{+} v \bar{v}$. Owing to the high beam-energy and high beam intensity, a long decay volume and a hermetic detector coverage, NA62 also has the opportunity to directly search for a plethora of hidden-sector particles, both in visible or invisible final states. We will detail on status and prospects of these searches, with special focus on those that can be performed with the experiment operated in beam-dump mode.
\end{abstract}

EPS-HEP 2017, European Physical Society conference on High Energy Physics

5-12 July 2017

Venice, Italy

* Speaker.

$\dagger^{\dagger}$ on behalf of the NA62 Collaboration:R. Aliberti, F. Ambrosino, R. Ammendola, B. Angelucci, A. Antonelli, G. Anzivino, R. Arcidiacono, M. Barbanera, A. Biagioni, L. Bician, C. Biino, A. Bizzeti, T. Blazek, B. Bloch-Devaux, V. Bonaiuto, M. Boretto, M. Bragadireanu, D. Britton, F. Brizioli, M.B. Brunetti, D. Bryman, F. Bucci, T. Capussela, A. Ceccucci, P. Cenci, V. Cerny, C. Cerri, B. Checcucci, A. Conovaloff, P. Cooper, E. Cortina Gil, M. Corvino, F. Costantini, A. Cotta Ramusino, D. Coward, G. D’Agostini, J. Dainton, P. Dalpiaz, H. Danielsson, N. De Simone, D. Di Filippo, L. Di Lella, N. Doble, B. Dobrich, F. Duval, V. Duk, J. Engelfried, T. Enik, N. Estrada-Tristan, V. Falaleev, R. Fantechi, V. Fascianelli, L. Federici, S. Fedotov, A. Filippi, M. Fiorini, J. Fry, J. Fu, A. Fucci, L. Fulton, E. Gamberini, L. Gatignon, G. Georgiev, S. Ghinescu, A. Gianoli, M. Giorgi, S. Giudici, F. Gonnella, E. Goudzovski, C. Graham, R. Guida, E. Gushchin, F. Hahn, H. Heath, T. Husek, O. Hutanu, D. Hutchcroft, L. Iacobuzio, E. Iacopini, E. Imbergamo, B. Jenninger, K. Kampf, V. Kekelidze, S. Kholodenko, G. Khoriauli, A. Khotyantsev, A. Kleimenova, A. Korotkova, M. Koval, V. Kozhuharov, Z. Kucerova, Y. Kudenko, J. Kunze, V. Kurochka, V.Kurshetsov, G. Lanfranchi, G. Lamanna, G. Latino, P. Laycock, C. Lazzeroni, M. Lenti, G. Lehmann Miotto, E. Leonardi, P. Lichard, L. Litov, R. Lollini, D. Lomidze, A. Lonardo, P. Lubrano, M. Lupi, N. Lurkin, D. Madigozhin, I. Mannelli, G. Mannocchi, A. Mapelli, F. Marchetto, R. Marchevski, S. Martellotti, P. Massarotti, K. Massri, E. Maurice, M. Medvedeva, A. Mefodev, E. Menichetti, E. Migliore, E. Minucci, M. Mirra, M. Misheva, N. Molokanova, M. Moulson, S. Movchan, M. Napolitano, I. Neri, F. Newson, A. Norton, M. Noy, T. Numao, V. Obraztsov, A. Ostankov, S. Padolski, R. Page, V. Palladino, C. Parkinson, E. Pedreschi, M. Pepe, M. Perrin-Terrin, L. Peruzzo, P. Petrov, F. Petrucci, R. Piandani, M. Piccini, J. Pinzino, I. Polenkevich, L. Pontisso, Yu. Potrebenikov, D. Protopopescu, M. Raggi, A. Romano, P. Rubin, G. Ruggiero, V. Ryjov, A. Salamon, C. Santoni, G. Saracino, F. Sargeni, V. Semenov, A. Sergi, A. Shaikhiev, S. Shkarovskiy, D. Soldi, V. Sougonyaev, M. Sozzi, T. Spadaro, F. Spinella, A. Sturgess, J. Swallow, S. Trilov, P. Valente, B. Velghe, S. Venditti, P. Vicini, R. Volpe, M. Vormstein, H. Wahl, R. Wanke, B. Wrona, O. Yushchenko, M. Zamkovsky, A. Zinchenko. 


\section{Introduction}

After the discovery of the Higgs boson at the LHC [1, 2], the experimental validation of the Standard Model (SM) is now completed. An additional LHC result of great importance is that a large parameter space has been explored and no New Physics (NP) was found, so far. These results, together with the constraints on NP from flavour phenomenology and the absence so far of charged lepton flavour violation processes allow us today to seriously consider the possibility that the SM could hold well beyond the electroweak (EW) scale $[3,4,5]$.

However there are experimental facts today that call for an explanation: what is the nature of Dark Matter (DM), why the Universe is dominated by matter, which is the origin of the neutrino masses. So far the experimental efforts have been concentrated on the discovery of new particles with masses at or above the EW scale with sizeable couplings with SM particles. Another viable possibility, largely unexplored, is that particles responsible of the still unexplained phenomena beyond the SM are below the EW scale and have not been detected because they interact very feebly with the SM particles [6].

The minimal set of particles needed to explain these phenomena are three right-handed singlet neutrinos which generate Majorana's masses and oscillations for the three SM neutrinos through the see-saw mechanism $[7,8,9,10]$ and are responsible of the baryogenesis through leptogenesis [11, 12], and some DM candidate which, if in the sub-GeV mass range, requires light mediators to deplete the overabundance in the early Universe [13, 14, 15, 16, 17, 18].

These hidden sector mediators are light, long-lived, feebly-interacting particles and mix with SM fields that do not carry electromagnetic charge, like the Higgs and the Z-bosons, the photon and the neutrinos. A vibrant and lively search for HNLs, light DM and hidden-sector mediators in the $\mathrm{MeV}-\mathrm{GeV}$ range is currently ongoing world-wide [19]. The NA62 experiment [20], operated both in beam and in beam-dump mode, will be one of the players in this field in the coming years.

\section{The beam and detector of the NA62 experiment}

A measurement of the branching ratio (BR) of the $K^{+} \rightarrow \pi^{+} v \bar{v}$ decay with $10 \%$ precision at least is the main goal of the NA62 experiment which is currently taking data at the CERN SPS. In order to achieve this goal the experiment needs to collect about $10^{13}$ kaon decays, $o\left(\right.$ few $\times 10^{12}$ ) have been already collected in the current run. Figure 1 shows the layout of the experiment.

In the NA62 beam line, the primary protons impinge on a $400 \mathrm{~mm}$ long, $2 \mathrm{~mm}$ diameter cylindrical Beryllium $(\mathrm{Be})$ target used to produce a secondary positively charged hadron beam of 75 $\mathrm{GeV} / \mathrm{c}$ momentum that reaches the $120 \mathrm{~m}$ long, $2 \mathrm{~m}$ diameter, in-vacuum decay volume, $100 \mathrm{~m}$ downstream of the target. A Cherenkov counter (KTAG) filled with $N_{2}$ along the beam line identifies and timestamps kaons, which are about $6 \%$ of the hadron beam. Three silicon pixel stations (Gigatracker, GTK) measure the momentum and the time of all the particles in the beam at a rate of $750 \mathrm{MHz}$. A guard ring detector (CHANTI) tags hadronic interactions in the last GTK station at the entrance of the decay volume. Large angle electromagnetic calorimeters (LAV) made of lead glass blocks surround the decay vessel can be used to veto particles up to $50 \mathrm{mrad}$. A magnetic spectrometer made of straw tubes in vacuum measures the momentum of the charged particles. A $17 \mathrm{~m}$ long RICH counter filled with Neon separates $\pi, \mu$ and $e$ up to $40 \mathrm{GeV} / \mathrm{c}$. The time of charged 


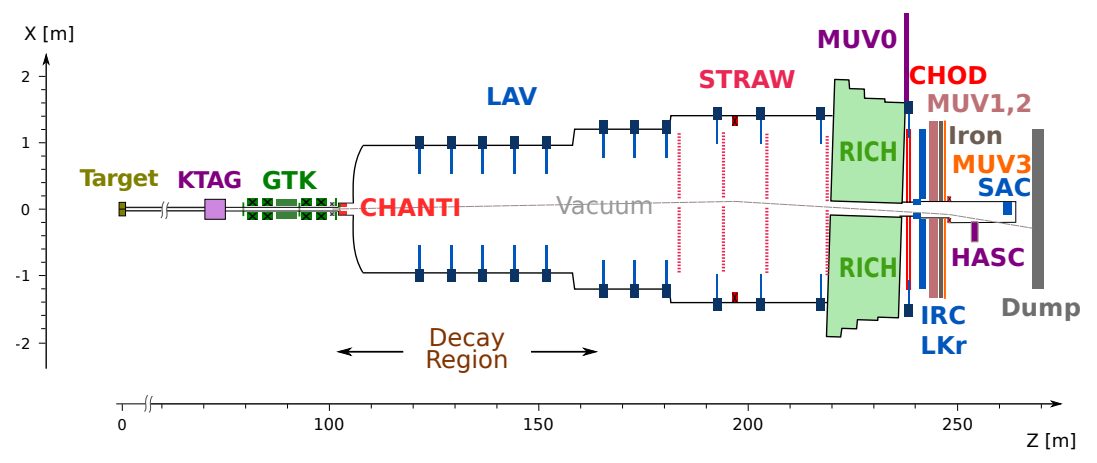

Figure 1: Layout of the NA62 experiment.

particles is measured both by the RICH and by scintillator hodoscopes (CHOD and NA48-CHOD) placed downstream to the RICH. The electromagnetic calorimeter filled with liquid kripton (LKr) covers the forward region and complements the RICH for the particle identification. A shashlik small-angle calorimeter (IRC) in front of $\mathrm{LKr}$ detects $\gamma$ directed on the inner edges of the $\mathrm{LKr}$ hole around the beam axis. The hadronic calorimeter made of two modules of iron-scintillator sandwiches (MUV1 and MUV2) provides further $\pi-\mu$ separation based on hadronic energy. A fast scintillator array (MUV3) identifies muons with sub-nanosecond time resolution. A shashlik calorimeter (SAC) placed on the beam axis downstream of a dipole magnet bending off-axis the beam at the end of the NA62 detector, detects $\gamma$ down to zero angle. A multi-level trigger architecture is used. The hardware-based level-0 trigger uses timing information from CHOD, RICH and MUV3, and calorimetric variables from electromagnetic and hadronic calorimeters. Higherlevel software-based trigger requirements are based on variables from KTAG, LAV and magnetic spectrometer.

\section{Search for hidden particles with NA62 operated in beam mode}

A large variety of searches for hidden sector particles can be performed at NA62 using kaon decays. Examples are: search for a Dark Scalar (S) or axion-like particle (ALP) from the decay $K^{+} \rightarrow \pi^{+} S / A L P s$, search for HNLs in the decays $K^{+} \rightarrow \mu^{+} / e^{+} v_{\mu / e}$, search for a Dark Photon $A^{\prime}$ in the decay $K^{+} \rightarrow \pi^{+} \pi^{0}, \pi^{0} \rightarrow A^{\prime} \gamma$, with $A^{\prime}$ decaying to invisible final states. The searches for HNLs in $K^{+} \rightarrow \mu^{+} v_{\mu}$ and $K^{+} \rightarrow e^{+} v_{e}$ decays has been performed using data sets collected by NA62 in 2007 [21] and 2015, respectively, and are described in M. Koval's report [22] in these proceedings.

A search for $A^{\prime}$ decaying into invisible final states has been performed using the decay $K^{+} \rightarrow$ $\pi^{+} \pi^{0}$ with $\pi^{0} \rightarrow \gamma A^{\prime}$ and $A^{\prime}$ escaping the detection. About $1.5 \times 10^{10} K^{+}$decays from the sample used for the $K^{+} \rightarrow \pi^{+} v \bar{v}$ analysis have been analyzed. This corresponds to about $5 \%$ of the dataset collected in 2016. Events with a single downstream track reconstructed in the straw spectrometer and matching in time and space energy depositions in calorimeters and hits in the NA48-CHOD are selected. The RICH and the calorimeters' system identify the downstream track as a pion, with a $\mu \rightarrow \pi$ misidentification of $o\left(10^{-7}\right)$. The missing mass obtained from the momentum of the 
downstream and GTK tracks is required to be around the $\pi^{0}$ mass peak. The selected sample is normalized to a sample of $K^{+} \rightarrow \pi^{+} \pi^{0}$ events with two photons reconstructed. Given the kaon, pion and photon momenta, the squared missing mass $M_{\text {mass }}^{2}=\left(P_{K}-P_{\pi}-P_{\gamma}\right)^{2}$ is expected to peak around the $A^{\prime}$ mass for the $\pi^{0} \rightarrow \gamma A^{\prime}$ decay and around zero for the background process $\pi^{0} \rightarrow \gamma \gamma$ decays with one photon undetected. The peak of the background events has a width left-right symmetric, hence a data-driven background estimate based on the tail with negative missing mass values is used. For each $A^{\prime}$ mass, the signal region is defined as a 1.5 -standard deviation range around the expected invariant mass peak. Frequentistic 90\% CL intervals have been determined, taking into account the uncertainties of signal efficiency from MC determination, and the statistical uncertainties of data counts and background expectations. No statistically significant excess has been detected and upper limits have been computed on the number of signal events. The $90 \%$ confidence level (CL) exclusion limit on the kinetic mixing parameter $\varepsilon$ versus the mass of the dark photon $m_{A^{\prime}}$ is shown in Figure 2 together with the current limits from BaBar [23], NA64 [24] and E949 [25] experiments, and the region of the parameters that could explain the current $(g-2)_{\mu}$ anomaly or that excluded by the agreement of the anomalous magnetic moment of the electron $(g-2)_{e}$ with the expectations.

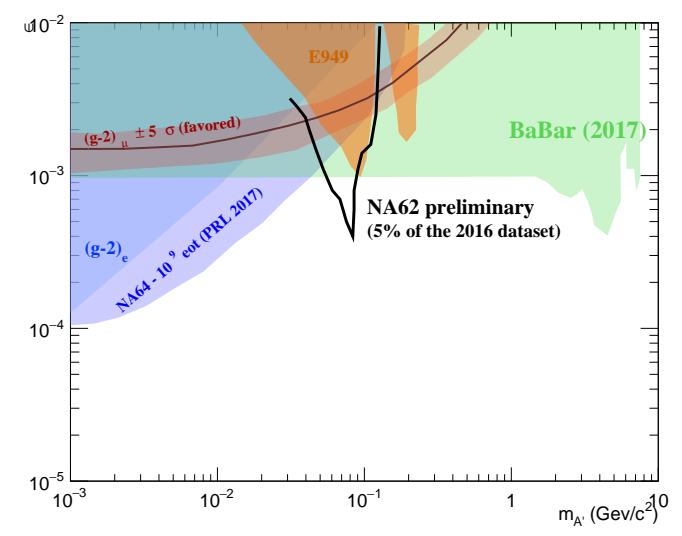

Figure 2: $90 \%$ CL exclusion limit in the $\varepsilon$ versun $m_{A^{\prime}}$ plane for $\pi^{0} \rightarrow \gamma A^{\prime}$ events with $A^{\prime} \rightarrow$ invisible final states. The limits from BaBar [23], NA64 [24] and E949 [25] are also shown together with the region of the parameters that could explain $(g-2)_{\mu}$ anomaly (red band) and the region excluded by the agreement of the anomalous magnetic moment of the electron $(g-2)_{e}$ with the expectations (dark blue shaded area).

\section{Search for hidden particles with NA62 operated in beam-dump mode}

Feebly-interacting long-lived particles can be originated also by the decay of beauty and charm hadrons, and by photons produced in the interaction of protons with a dump. Their couplings to SM particles are very suppressed leading to expected production rates of $10^{-10}$ or less.

Since the charm and beauty cross-sections steeply increase with the energy [26]-[28], a highintensity, high-energy proton beam is required to improve over the current results [29]: the 400 $\mathrm{GeV} / \mathrm{c}$ primary proton beam line serving the NA62 experiment with a nominal intensity of $3 \times$ 
$10^{12}$ protons per pulse, with pulses $4.8 \mathrm{sec}$ long, can produce high intensity fluxes of beauty and charm hadrons and photons through the interactions of protons on a dump. To operate the NA62 experiment in dump-mode, the $B e$ target can be pulled up and the primary proton beam can be sent directly onto the $\mathrm{Cu}-\mathrm{Fe}$ based NA62 collimators (TAXes) that act as a hadron stopper $20 \mathrm{~m}$ downstream of the $B e$ target. This operation can be done with the current beam setup easily, quickly ( 15 minutes) and in a fully reversible manner. In this configuration, about $2 \times 10^{15} \mathrm{D}$-mesons and $\sim 10^{11}$ b-hadrons would be produced with $\sim 10^{18}$ protons-on-target (pot), which correspond to $\sim 80$ days of data taking at the nominal NA62 beam intensity.

The experimental signature of hidden sector decays into SM particles is two or more tracks or two photons originating from the same point of the decay volume and nothing else. The experimental challenge is to disentangle the signal from backgrounds that mimick the same signature.

Figure 3 shows the expected NA62 sensitivity with $10^{18}$ pot for the interaction strength versus mass for Heavy Neutral Leptons (HNLs) for the three scenarios described in Refs. [30, 31]. Other shaded areas are excluded regions by past experiments and dotted lines are expected sensitivities of future experiments [19, 29]. For the HNLs, the allowed parameters range is defined by the seesaw mechanism and Big Bang Nucleo-synthesis (BBN) lines [32, 33], and the (model-dependent) Baryon Asymmetry of the Universe (BAU) line [34]. The sensitivity curves assume to detect all 2-track final states with zero background and include the geometrical acceptance and the trigger efficiency. The selection efficiency is assumed to be $100 \%$, however the inclusion of the selection efficiencies is expected to worsen the limits by $20 \%$ at most. The sensitivities achievable with $10^{18}$ pot for a vector mediator (Dark Photon) decaying to dimuon final states, for a scalar mediator (Dark Scalar) decaying to $e^{+} e^{-}, \mu^{+} \mu^{-}, \pi^{+} \pi^{-}, K^{+} K^{-}$final states, and for an Axion Like Particle (ALP) decaying to 2-photon final states [35] are shown in Figures 4 left, center and right, respectively. In all plots the shaded areas are excluded regions by past experiments and dotted lines are expected sensitivities of future experiments $[19,29,36]$. In all cases the effect of the geometrical acceptance and the trigger efficiency is included and zero-background is assumed. The validity of the last assumption is discussed in Section 5.
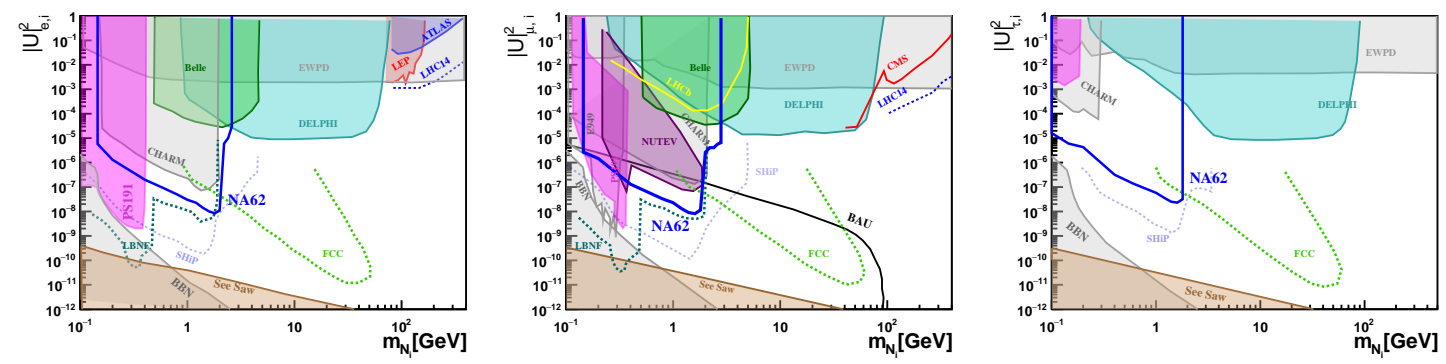

Figure 3: NA62 sensitivity (90\% CL exclusion limit) for $10^{18}$ pot in the coupling versus mass plane for HNLs originated by the dump (blue solid lines) for the ratio of coupling parameters $U_{e}^{2}: U_{\mu}^{2}: U_{\tau}^{2}=1: 16: 3.8$ (left), $52: 1: 1$ (center) and $0.061: 1: 4.3$ (right), as described in Refs. [30, 31]. The sensitivity below the kaon mass comes mostly from kaon experiments, as, for example, in Ref. [37]. 

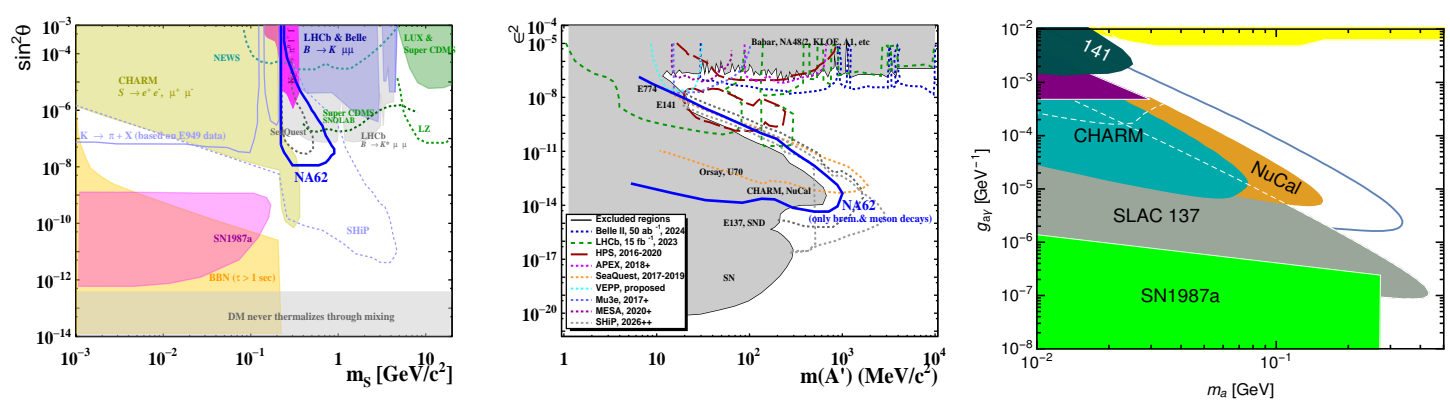

Figure 4: NA62 sensitivity (90\% CL exclusion limit) for $10^{18}$ pot in the coupling versus mass plane for Dark Scalar (left, blue solid line), Dark Photon (center, blue solid line), and ALPS (right, blue solid line) originated by the dump.

\section{Analysis of the background of NA62 operated in beam-dump mode}

The sensitivity to hidden sector particles can be greatly spoiled by the presence of background. In fact, the proton interactions on the dump, along with the signals, give rise to a copious direct production of short-lived resonances, and pions and kaons. While the length of the dump $\left(\sim 22 \lambda_{I}\right)$ is sufficient to absorb the hadrons and the electromagnetic radiation, the decays of pions, kaons and short-lived resonances result in a large flux of muons and neutrinos, which are the major sources of background in the apparatus. About $18 \mathrm{kHz}$ of muons have been measured at $40 \%$ nominal intensity in the NA62 acceptance in a $o(10)$ hours long run taken in dump mode in November 2016, where about $2 \times 10^{15}$ pot were recorded, and a preliminary study of the background rates and topologies has been performed.

A simple selection has been applied to this sample to search for generic 2-track final states, requiring good quality tracks and 2-track vertices, no further activity in time with the 2-track candidates, no additional track close to the selected vertices, and in-time coincidence of the two tracks at the NA48-CHOD. The reconstructed 2-track vertex is required to lie in the interval $115<z<180$ $\mathrm{m}$. Particle identification information has been used to study the background topologies. The opposite-charge 2-track sample is the dangerous component as it can mimick a signal signature: it comprises random combinations of tracks from the muon halo and muon inelastic interactions in the last $\lambda_{I}$ of the final collimator of the NA62 beam line, with one or no muons in the final state. In this category, the recontructed vertices are mostly concentrated at the beginning of the fiducial volume and the two tracks are mostly coincident in time.

Overall 28 events of the opposite-charge category are left after the selection. A powerful handle to further reduce the background while searching for fully reconstructed signal final states (eg: $H N L \rightarrow \pi^{ \pm} l^{\mp}$ with $l=\mu, e$; Dark Photon or Dark scalar $\rightarrow f^{+} f^{-}$, with $f=e, \mu, \pi, K$ ) is to require the events to point backwards to the dump: as expected, all the events from the analyzed data sample do not satisfy this condition and can be removed by a mild cut in impact parameter. However, also most of the HNLs' decays do not point backwards to the dump, as they contain along with the 2-tracks, also photons and neutrinos in the final state that are not detected (eg: $H N L \rightarrow \pi^{ \pm} \tau^{\mp} ; H N L \rightarrow l^{+} l^{-} v$ or $\rho^{ \pm} l^{\mp}$, with $l=e, \mu, \tau$ and $\rho^{ \pm} \rightarrow \pi^{ \pm} \pi^{0}$ and $\tau \rightarrow 1$ prong X). Generic models of asymmetric Dark Matter [38], with $A \rightarrow \chi_{1} \chi_{2}$, and $\chi_{2} \rightarrow f^{+} f^{-}$also contain 
partially reconstructed final states.

The extrapolation of the residual background tracks at the entrance of the decay vessel shows that they are all concentrated in a zone not covered by the current apparatus. The addition of an Upstream Veto in front of the fiducial volume with $10^{-4}$ detection inefficiency and 200-300 ps time resolution would allow to reduce the background for 2-track final states to a few events for $10^{18}$ pot allowing NA62 to reach the ultimate sensitivity for this number of protons on target for a wide variety of hidden sector models.

\section{Conclusions}

NA62 is successfully running in the North Area of the CERN SPS with the main goal of measuring the $B R\left(K^{+} \rightarrow \pi^{+} v \bar{v}\right)$ with $10 \%$ accuracy. Data collected so far [39] show that an accuracy of $\sim 20 \%$ can be reached in the current run (2016-2018). Owing to the high beam energy and high beam intensity, the long decay volume and the hermetic detector coverage, NA62 also has the opportunity to directly search for a plethora of hidden-sector particles, both in visible or invisible final states.

A large variety of searches for hidden sector particles can be already performed in beam mode using kaon decays and an even broader and richer physics programme can be proposed for the 2021-2023 data taking, including $\sim 10^{18}$ pot ( $~ 80$ days) in dump-mode while the large majority $(\sim 85 \%)$ of the beam time will be dedicated to kaon physics. Preliminary studies with data taken in beam and beam-dump modes show that the background can be kept under control for most of the decay channels with 2-track final states when an Upstream Veto is added to the current setup.

\section{References}

[1] ATLAS Collaboration, G. Aad et al., Phys. Lett. B716 (2012) 1.

[2] CMS Collaboration, S. Chatrchyan et al., Phys.Lett. B716 (2012) 30.

[3] G. Degrassi et al., JHEP 08 (2012) 098.

[4] D. Buttazzo et al., JHEP 12 (2013) 089.

[5] F. Bezrukov et al., JHEP 10 (2012) 140.

[6] R. Essig, J. A. Jaros, W. Wester, et al., arXiv:1311.0029.

[7] P. Minkowski, Phys. Lett. B67 (1977) 421.

[8] T. Yanagida, Prog. Theor. Phys. 64 (1980) 1103.

[9] M. Gell-Mann, P. Ramond, and R. Slansky, Rev. Mod. Phys 50 (1978) 721.

[10] R. N. Mohapatra and G. Senjanovic, Phys. Rev. Lett. 44 (1980) 912.

[11] M. Fukugita and T. Yanagida, Phys. Lett. B174 (1986) 45.

[12] For reviews see, for example: W. Buchmuller, R.D. Peccei and T. Yanagida, Ann. Rev. Nucl. Part. Sci. 55, 311 (2005), arXiv:0502169 [hep-ph]; S. Blanchet and P. Di Bari, New J. Phys. 14, 125012 (2012); T. Hambye, New J. Phys. 14, 125014 (2012).

[13] C. Boehm, T. Ensslin, and J. Silk, J. Phys. G30, 279 (2004). 
[14] C. Boehm and P. Fayet, Nucl. Phys. B683, 219 (2004).

[15] M. Pospelov, A. Ritz, and M. B. Voloshin, Phys. Lett. B662, 53 (2008).

[16] M. Pospelov, Phys. Rev. D80, (2009) 095002.

[17] N. Arkani-Hamed, D. P. Finkbeiner, T. R. Slatyer, and N. Weiner, Phys. Rev. D79, (2009) 015014.

[18] M. Pospelov and A. Ritz, Phys. Lett. B671, (2009) 391.

[19] for an updated review of the running and planned experiments see Dark Sector 2016 Workshop: Community Report, Workshop on Dark Sectors, April 2016, SLAC (US), arXiv:1608.08632.

[20] NA62 Collaboration, JINST 12 (2017) no.05, P05025.

[21] NA62 collaboration, Phys. Lett. B772 (2017) 712.

[22] M. Koval on behalf of the NA62 collaboration, New limits on heavy neutrino from NA62, talk at EPS-HEP Conference, Venice (Italy), July 2017.

[23] BaBar collaboration (J. P. Lees et al.), Phys. Rev. Lett. 119 (2017) 131804.

[24] NA64 collaboration (D. Banerjee et al.), Phys. Rev. Lett. 118 (2017) no.1, 011082.

[25] E949 collaboration (A. V. Artamonov et al.), Phys. Rev. D79 (2009) 092004.

[26] C. Lourenco and H. Wohri, Phys. Rept. 433, (2006) 127.

[27] H. Abramowicz et al., JHEP 1309, 058, (2013).

[28] K. Olive et al., Chin. Phys. C38 (2014) 090001.

[29] For a recent review see S. Alekhin et al., Rept. Prog. Phys. 79 (2016) no.12, 124201, and References therein.

[30] T. Asaka, S. Blanchet, and M. Shaposhnikov, Phys. Lett. B631 (2005) 151.

[31] T. Asaka and M. Shaposhnikov, Phys. Lett. B620 (2005) 17.

[32] A. D. Dolgov, S. H. Hansen, G. Raffelt and D. V. Semikoz, Nucl. Phys. B 590 (2000) 562.

[33] O. Ruchayskiy and A. Ivashko, JCAP 10 (2012) 014.

[34] M. Canetti and M. Shaposhnikov, JCAP 1009 (2010) 001.

[35] B. Dobrich et al., JHEP 02 (2016) 018.

[36] A. Atre, T. Han, S. Pascoli and B. Zhang, JHEP 0905, (2009) 030.

[37] E949 collaboration (A. V. Artamonov et al.), Phys. Rev. D91 (2015) no.5 052001. Erratum in Phys.Rev. D91 (2015) no.5, 059903.

[38] E. Izaguirre et al., Phys. Rev. D96 (2017) no.5 055007.

[39] NA62 Status Report, CERN-SPSC-2017-013/SPSC-SR-208. 